\title{
Conceptualización de atributos de género en jóvenes universitarios del norte de México
}

\section{Conceptualization of gender attributes in university students from northern Mexico}

\author{
Elba Abril Valdez, María José Cubillas Rodríguez, \\ Sandra Elvia Ibáñez Domínguez y Rosario Román Pérez ${ }^{l}$
}

\begin{abstract}
RESUMEN
El objetivo del estudio fue explorar la conceptualización semántica que estudiantes universitarios del norte de México tienen sobre las palabras hombre-mujer y femenino-masculino. Se analizó la información de 1,514 estudiantes seleccionados al azar de una universidad pública. La técnica utilizada fue la asociación de palabras, que permitió documentar las asignaciones culturales a través de la organización conceptual. Los resultados muestran que ambos sexos relacionan estas palabras con atributos asignados tradicionalmente a cada sexo. Tal persistencia indica la necesidad de cuestionar y teorizar sobre esas ideas estereotipadas en una población educada y aparentemente más permeada por los cambios sociales sobre la igualdad y la equidad de género.
\end{abstract}

Palabras clave: Género; Hombre; Mujer; Masculino; Femenino; Concepto.

\begin{abstract}
ABSTACT
The present study explored the semantic conceptualization of university students in northern Mexico over the words man-woman and female-male. The main technique involved word association. This procedure allowed for data collection and documenting cultural assignments through conceptual organization. The results show that both sexes relate these words with traditional attributes usually assigned to each sex. Such homogeneous persistence seems to indicate the need to question and theorize about these traditional ideas in an educated population, supposedly permeated by recent social changes on gender equality and equity.
\end{abstract}

Key words: Gender; Man; Woman; Male; Female; Concept.

\section{INTRODUCCIÓN}

$\mathrm{L}$

as personas aprenden a comportarse como hombres y mujeres en función de representaciones culturales de género que permean el carácter de las relaciones que se mantienen en diferentes escenarios sociales, tales como la familia, la escuela y los pares, entre otros. Así, el género establece los referentes culturales que cada sexo reconoce y asume. Tales representaciones son internalizadas por los sujetos que forman parte de la sociedad, estructurando y configurando con ello formas de ser, actuar y pensar de unos y otras sobre la realidad, de tal manera que se van marcando y reconociendo ciertos estereotipos del "deber ser" de cada sexo (Lamas, 2002; Scott, 2003).

${ }^{1}$ Centro de Investigación en Alimentación y Desarrollo, A.C., Carretera a La Victoria km. 0.6, 83304 Hermosillo, Sonora, México, direcciones electrónicas: abril@ciad.mx,mjcubillas@ciad.mx,dominguez@ciad.mx y rroman@ciad.mx. Artículo recibido el23 de octubre y aceptado el 13 de diciembre de 2017.

Citación: Valdez, E.A., Cubillas R., M.J., Ibáñez D., S.E. y Román P., R. (2018). Conceptualización de atributos de género en jóvenes universitarios del norte de México. Psicología y Salud, 28(2), 229-238. 
La familia es la principal transmisora de las actitudes, valores, comportamientos y prácticas que conforman la identidad humana social y cultural de las personas y la que establece la regla de cómo comportarse, roles que se asumen en función de las relaciones consanguíneas, a partir de las expectativas y exigencias atribuibles al género, mismas que determinan y afectan el perfil y la identidad de cada integrante del grupo. La institución familiar es un ámbito de interacción y convivencia en el que se gestan relaciones asimétricas entre sus integrantes (hombres o mujeres, adultos o jóvenes) (Lamas, 2002).

Es, pues, en el proceso de socialización en el seno familiar cuando se establecen las expectativas de lo que los hombres y las mujeres deben ser como sujetos masculinos y femeninos, y desempeñar por consiguiente los roles de género asignados. Así, el varón debe de proveer, ser racional, carecer de emotividad, seguro, viril, dominante, activo y tener otras características estereotipadas, como la independencia o el egoísmo, entre otras. A la mujer se le enseña a ser femenina, responsable de la felicidad de la pareja, de los hijos y de la familia; del manejo de las emociones, de la expresión de modales que demuestran fragilidad y delicadeza y otros muchos atributos que suponen dependencia, inseguridad, sentimentalismo, expresividad afectiva y emocional, ternura, sumisión, pasividad y debilidad (Lara, 1993; Maldonado y Segovia, 2010; Ortiz, 2004).

Tales concepciones sobre el "deber ser" de hombres y mujeres constituyen los estereotipos de género, y existe la presión social para que, independientemente del nivel educativo de las personas, satisfagan las expectativas sociales y familiares que los adscriben a espacios y actividades separadas.

La conceptualización de los términos hombre y mujer, femenino y masculino están colmados de estereotipos y dobles códigos. Según Díaz-Guerrero (1989), el significado connotativo de un concepto está compuesto por elementos afectivos y conocimientos que expresan la cultura y las ideas del universo que cada individuo tiene, así como por elementos compartidos por el grupo al que pertenece. Por lo tanto, es preciso reconocer la importancia del estudio y la comprensión de la terminología mencionada en el contexto cultural, pues la cosmovisión de un grupo constituye una forma particular de ver el mundo.

En Latinoamérica estudios realizados con jóvenes sugieren que tanto hombres como mujeres conceptualizan el deber ser de cada sexo con estereotipos reforzados socialmente y que los llevan a exhibir conductas y actitudes sobreentendidas y esperadas para ambos sexos (Petra, Talayero, Fouilloux y Díaz, 2000; Varela, 2012).

La sociedad mexicana, y particularmente la sonorense, se caracteriza por ser conservadora y orientada a mantener las tradiciones.

Datos del Instituto Nacional de Estadística y Geografía (INEGI) (2017) señalan que 58\% de hombres y mujeres están casados y viven en familias nucleares (que ascienden a 70\%), formadas por el padre, la madre y los hijos, siendo el primero el principal sustento del hogar en $73 \%$ de estos. Núñez (2013) observó en tres cohortes de hombres sonorenses algunos cambios en la conceptualización del género, la sexualidad y la reproducción, aunque persisten y coexisten con ellas algunos comportamientos tradicionales referidos a situaciones cotidianas tales como la crianza de los hijos, las tareas domésticas o las decisiones sobre la anticoncepción. Igualmente, Rocha y Díaz-Loving (2005) señalan que en la cultura mexicana el varón está ligado al prototipo del rol instrumental, que se manifiesta en las actividades productivas caracterizadas por la autonomía, la orientación al logro, la fuerza, el éxito y el rol de proveedor, en tanto que a la mujer se le vincula con actividades afectivas encaminadas al cuidado de los hijos, el hogar y la pareja.

Los roles y estereotipos asignados a cada sexo que determinan lo femenino y lo masculino están muy delimitados y arraigados en la mayoría de la gente. Sin embargo, hoy día los jóvenes tienen acceso a más información, e incluso participan en programas con perspectiva de género que se implementan en las instituciones de educación superior (IES). Por ejemplo, desde el periodo 2010-2013 se puso en práctica la política pública de "transversalizar" la visión de género en las instituciones públicas, incluyendo las IES, a fin de promover la incorporación de la perspectiva de género en las políticas, planes, programas y proyectos de las universidades. Por lo mismo, se señala que las IES deben contar con un pro- 
grama para difundir y fomentar entre el personal académico, el administrativo y el estudiantado una cultura apegada a la igualdad, la equidad y la diversidad, para así permear la rigidez de las estructuras académicas de esas instituciones. Se espera con ello promover una educación orientada hacia la igualdad, generando en los jóvenes nuevas formas de asumir el ser hombre y ser mujer, expresadas de algún modo a través de las conceptualizaciones que los definen.

Partiendo de lo anterior, el objetivo de este trabajo fue analizar la conceptualización semántica que estudiantes universitarios hacen de los términos hombre-mujer y femenino-masculino, a fin de explorar sus asignaciones culturales a través de la organización conceptual de los participantes en torno a estas cuatro palabras. Mediante la técnica de redes semánticas naturales (Reyes, 1993; Valdez, 2002) se analizaron dichos conceptos para explorar y ofrecer una explicación de la representación y la organización del conocimiento que los participantes tenían en la memoria. La información contenida en la misma se representa semánticamente con redes de conceptos en las que las palabras y los sucesos forman relaciones que, en conjunto, producen el significado. Tal análisis hace posible generar un sistema de información sobre ideas, actitudes y creencias, entre otras variables, de una población particular. Las redes semánticas posibilitan no solamente describir el conocimiento de los sujetos, sino que ponen en evidencia su cosmovisión ante un evento (estímulo) (Figueroa, González y Solís, 1981; Reyes, 1993; Valdez, 2002.)

\section{MÉTODO}

\section{Participantes}

Se trabajó con jóvenes estudiantes de una universidad pública ubicada en la ciudad de Hermosi1lo, en el estado de Sonora, en la frontera norte de México. La IES contaba al momento del estudio con una población inscrita de 21,316 alumnos, de los cuales 10,684 eran hombres y 10,632 mujeres. Los participantes se seleccionaron a través de un muestreo estratificado (Cochran, 1990), con un nivel de confianza de $95 \%$ y un margen de error de 0.05 . La muestra se conformó con 1,514 estudiantes distribuidos en las 32 licenciaturas que ofrece la universidad. La proporción de hombres y mujeres fue similar, con una ligera representación mayor de las mujeres: De los participantes, 51.9\% (786) fueron mujeres y 48.1\% (728) varones. La media de edad en ambos sexos fue de 20 años, con un rango de 17 a 29. La mayoría estuvo constituida por solteros (94.8\% hombres y $92.6 \%$ mujeres), y más mujeres que hombres $(6.8$ y $4.8 \%$, respectivamente) dijeron tener una relación estable de pareja, ya sea por estar casados o vivir en unión libre. El resto de los participantes dijeron estar separados o divorciados. De los varones, $4.3 \%$ dijo tener hijos, al igual que $6.4 \%$ de las mujeres; el promedio de hijos fue de 1.4, con un rango de 1 a 4 .

En cuanto al origen, la mayoría de los participantes procedía de Hermosillo; muy pocos provenían de otras localidades de dentro o de fuera del estado. En cuanto a su estado conyugal, los datos concuerdan con lo reportado para Sonora por el INEGI (2013); así, se pudo apreciar que los hombres y las mujeres del grupo de 15 a 29 años eran solteros en su mayoría, con $74.1 \%$ en el caso de los hombres y $65.3 \%$ en el de las mujeres. Igualmente, según el INEGI, en la referida entidad las mujeres son más las que están unidas en pareja o tienen hijos. Lo anterior muestra que las mujeres cumplen con el rol esperado socialmente al ser ellas quienes conforman una familia a más temprana edad. Poco más de la mitad dijo vivir con sus padres en familias nucleares $(51.3 \%$ varones y $55.9 \%$ mujeres); $14.7 \%$ de los varones y $13.4 \%$ de las mujeres vivían solamente con alguno de los padres, mismo porcentaje que se observa entre los que reportaron vivir con abuelos, tíos o hermanos. Más hombres (7.8\%) que mujeres (4.8\%) vivían solos; y $5.6 \%$ y $6.2 \%$, respectivamente, indicaron vivir en familias extensas o reconstruidas; el resto de los participantes dijo vivir con amigos o en casas de asistencia.

\section{Instrumento}

Para recabar la información se aplicó la técnica de asociación libre de palabras (Reyes, 1993). Así, se presentaron a los estudiantes cuatro palabras-estímulo: mujer y hombre, y masculino y femenino, para que los jóvenes evocaran términos que aso- 
ciaran con dichos conceptos. Igualmente, se recabó información sociodemográfica, como edad, semestre y tipo de familia.

\section{Procedimiento}

La participación de los estudiantes fue voluntaria y una vez obtenido el acuerdo de las autoridades de la IES. Antes de la aplicación se les informó el objetivo del estudio y se aseguró la confidencialidad de la información que proporcionaran, tras de lo cual expresaron su consentimiento informado. La aplicación se llevó a cabo de manera colectiva y en los horarios de clase. Se pidió a los participantes que anotaran tres términos que pensaran, sintieran o creyeran que, según su criterio, representaban el concepto presentado, dándole una connotación de mayor valor (peso semántico) al primero que anotaran, y así sucesivamente. La descripción de la información se presenta por sexo en el apartado de resultados.

\section{Plan de análisis}

Una vez obtenida la información, se capturaron todas las palabras y se jerarquizaron en una hoja de cálculo del programa Excel. Los valores se obtuvieron con base en la nomenclatura propuesta por Reyes (1993), que a su vez se deriva de la utilizada por Figueroa et al. (1981). Dichos valores son los más usuales y básicos, apropiados para conocer el significado de algún concepto en una cultura o grupo social determinado.

El peso semántico (PS) de cada una de las definidoras se obtiene a través de la suma de la ponderación de la frecuencia por la jerarquización asignada por el grupo, donde los 1 (la definidora más cercana al concepto, según el participante) se multiplican por diez, los 2 por nueve, los 3 por ocho, y así hasta completar el ordenamiento de las definidoras anotadas por los participantes. El tamaño de la red (TR) se obtiene contando el número de definidoras mencionadas, y la distancia semántica cuantitativa (DSC) indica en forma de porcentaje la distancia semántica que hay entre las diferentes palabras definidoras que conforman el conjunto de la red. En el núcleo de la red (NR) se hallan las diez palabras definidoras con el mayor PS. Las redes semánticas naturales hacen posible cuantifi- car detalladamente las diferencias individuales y grupales. También permiten la elaboración de un sistema de información acerca de ideas, actitudes, creencias y demás.

\section{RESULTADOS}

\section{Los conceptos hombre-mujer}

En la Tabla 1 se observa que para el estímulo hombre los varones proporcionaron un total de 154 definidoras y las mujeres de 142. El concepto hombre fue definido principalmente por los participantes con la palabra "fuerte". De entre las quince palabras descriptoras más mencionadas por hombres y mujeres se pueden observar semejanzas en $70 \%$ de ellas; es decir, ambos sexos definen hombre con atributos culturalmente asignados para los varones, como "trabajador", "padre", "proveedor", "responsable", "duro" o "inteligente", entre otros.

Vale la pena subrayar que en el NR del concepto hombre aparecen palabras con una clara connotación cultural de género. Hombres y mujeres asocian este concepto con las dos primeras definidoras: "fuerte" y "trabajador". Aparecen además en la red de ambos sexos los términos "responsable", "proveedor", "protector" y "jefe de familia", términos que aluden al papel productivo adjudicado culturalmente a los varones. La elección que hacen las mujeres de palabras asociadas con dicho concepto implican atributos como la fuerza, el poder y el éxito que garanticen para ellas una buena proveeduría y que ubican a los varones en una elevada posición jerárquica. Los hombres enlistan entre las definidoras la palabra "líder", y en el caso de las mujeres aparecen las de "guapo", "amor" y "caballero", conceptos asociados tradicionalmente al amor idealizado y romántico. Tales conceptos desempeñan un importante papel en el sistema de creencias que la gente comparte. El trabajo aparece como el sostén de la identidad de los hombres, quienes siguen representando la racionalidad, la separación de ámbitos que determina que su identidad social se construya justamente sobre el trabajo, la proveeduría y la función como cabeza de la familia, independientemente del tipo de pareja y de familia (Lamas, 1996), lo que muestra el peso de una cultura androcéntrica. 
Tabla 1. Definidoras para el concepto hombre.

\begin{tabular}{|l|r|r|l|r|r|}
\hline \multirow{2}{*}{ Definidora } & \multicolumn{2}{|c|}{ Hombres } & \multirow{2}{*}{ Definidora } & \multicolumn{2}{c|}{ Mujeres } \\
\cline { 2 - 3 } \cline { 5 - 6 } & \multicolumn{1}{c|}{ PS } & DSC (\%) & & PS & DSC (\%) \\
\hline Fuerte & 1694 & 100.00 & Fuerte & 2575 & 100.00 \\
\hline Trabajador & 1622 & 95.75 & Trabajador & 1531 & 59.46 \\
\hline Amigo & 1238 & 73.08 & Macho & 1245 & 48.35 \\
\hline Padre & 1073 & 63.34 & Padre & 1219 & 47.34 \\
\hline Macho & 705 & 41.62 & Proveedor & 757 & 29.40 \\
\hline Responsable & 597 & 35.24 & Responsable & 535 & 20.78 \\
\hline Proveedor & 399 & 23.55 & Protector & 453 & 17.59 \\
\hline Duro & 359 & 21.19 & Pareja & 401 & 15.57 \\
\hline Hermano & 358 & 21.13 & Guapo & 396 & 15.38 \\
\hline Protector & 238 & 14.05 & Inteligente & 372 & 14.45 \\
\hline Humano & 231 & 13.64 & Amor & 333 & 12.93 \\
\hline Jefe de familia & 224 & 13.22 & Jefe de familia & 300 & 11.65 \\
\hline Líder & 198 & 11.69 & Amigo & 285 & 11.07 \\
\hline Respeto & 182 & 10.74 & Duro & 239 & 9.28 \\
\hline Inteligente & 160 & 9.45 & Caballero & 217 & 8.43 \\
\hline TR & 154 & & TR & 142 & \\
\hline
\end{tabular}

Abrev.: PS = Peso semántico, $\mathbf{D S C}=$ Distancia semántica cuantitativa, $\mathbf{T R}=$ Tamaño de la red.

Una de las formas encubiertas más comunes del poder de los hombres sobre las mujeres es esa noción de ser el proveedor y protector, el jefe, el macho, el responsable. Los hombres han sido los encargados tradicionalmente de la protección del grupo; el hombre joven asume más tarde ese rol de protector, por lo que es visto por las mujeres como el príncipe azul.
Como se observa en la Tabla 2, para el estímulo mujer los varones enunciaron un total de 185 definidoras y las mujeres 201; la palabra "bonita" aparece encabezando la red en el caso de los hombres, contrariamente a las mujeres, que la definieron sobre todo con la palabra "fuerte".

Tabla 2. Definidoras para el concepto mujer.

\begin{tabular}{|l|r|r|l|r|c|}
\hline \multirow{2}{*}{ Definidora } & \multicolumn{2}{|c|}{ Hombres } & Definidora & \multicolumn{2}{c|}{ Mujeres } \\
\cline { 2 - 6 } & \multicolumn{1}{|c|}{ PS } & DSC (\%) & & \multicolumn{1}{c|}{ PS } & DSC (\%) \\
\hline Bonita & 2,149 & 100.00 & Fuerte & 1,791 & 100.00 \\
\hline Madre & 1,447 & 67.33 & Madre & 1,629 & 90.95 \\
\hline Pareja & 1,035 & 48.16 & Bonita & 1,473 & 82.24 \\
\hline Amor & 1,012 & 47.09 & Amor & 880 & 49.13 \\
\hline Delicada & 505 & 23.50 & Femenina & 745 & 41.60 \\
\hline Amiga & 485 & 22.57 & Inteligente & 656 & 36.63 \\
\hline Sensible & 442 & 20.57 & Trabajadora & 613 & 34.23 \\
\hline Sexo & 399 & 18.57 & Sensible & 610 & 34.06 \\
\hline Inteligente & 394 & 18.33 & Procreación & 531 & 29.65 \\
\hline Femenina & 358 & 16.66 & Delicada & 505 & 28.20 \\
\hline Procreación & 356 & 16.57 & Responsable & 471 & 26.30 \\
\hline Ama de casa & 346 & 16.10 & Ama de casa & 373 & 20.83 \\
\hline Respeto & 338 & 15.73 & Respeto & 311 & 17.36 \\
\hline Género & 314 & 14.61 & Amiga & 304 & 16.97 \\
\hline Fuerte & 271 & 12.61 & Luchona & 294 & 16.42 \\
\hline TR & 185 & & TR & 210 & \\
\hline
\end{tabular}

Abrev: $:$ PS $=$ Peso semántico, $\mathbf{D S C}=$ Distancia semántica cuantitativa, $\mathbf{T R}=$ Tamaño de la red. 
De entre las quince palabras descriptoras más mencionadas por hombres y mujeres se pueden observar semejanzas en $80 \%$ de las definidoras, es decir, ambos sexos definen mujer con atributos que tradicionalmente se le asignan y que tienen que ver con aspectos afectivos o emocionales, como la maternidad, el cuidado y el amor. Ambos sexos colocan la palabra "madre" en segundo sitio, apareciendo el área de la maternidad ligada preponderantemente al rol de mujer. Con una clara connotación del rol de género, aparecen en ambos los términos "delicada", "sensible", "femenina" y "amor", entre otros. En este caso, la pareja y la maternidad son el sostén de la identidad de las mujeres. La madre sigue representando la sublimación de la emocionalidad: cuidar de otros, dar y entregarse, constituyendo así espacios sobre los que las mujeres edifican su identidad social, sin un espacio propio, acorde con lo señalado por Amurrio, Larrinaga, Usategui y Del Valle (2008).

Si bien, al igual que con el concepto hombre, comparten significados, la jerarquía de los valores según el grado de importancia fue diferente en mujeres y hombres. Por otro lado, es importante señalar que las mujeres utilizaron descriptores tales como "trabajadora", "luchona" y "responsable", atributos que, no obstante, son culturalmente asignados a los varones.

\section{Lo femenino y lo masculino}

Para el estímulo femenino los varones enlistaron un total de 136 definidoras y de 164 las mujeres. El concepto femenino fue definido por los jóvenes principalmente con la palabra "mujer". De entre las quince palabras descriptoras más mencionadas por hombres y mujeres se pueden observar semejanzas en $66.6 \%$ de ellas; esto es, ambos sexos definen femenino con los atributos o características que culturalmente se asignan a las mujeres, como delicadeza, belleza, dulzura o sensibilidad, entre otros. Tales conceptos denotan lo que se esperaría es propio de una mujer (Tabla 3).

Tabla 3. Definidoras para el concepto femenino.

\begin{tabular}{|l|r|r|l|r|c|}
\hline \multirow{2}{*}{ Definidora } & \multicolumn{2}{|c|}{ Hombres } & \multirow{2}{*}{ Definidora } & \multicolumn{2}{|c|}{ Mujeres } \\
\cline { 2 - 3 } & PS & DSC (\%) & & \multicolumn{1}{c|}{ PS } & DSC (\%) \\
\hline Mujer & 2,213 & 100.00 & Mujer & 1,964 & 100.00 \\
\hline Delicadeza & 1,309 & 59.15 & Delicadeza & 1,543 & 78.56 \\
\hline Belleza & 1,128 & 50.97 & Belleza & 1,281 & 65.22 \\
\hline Dulce & 365 & 16.49 & Accesorios & 983 & 50.05 \\
\hline Sensible & 257 & 11.61 & Dulce & 468 & 23.83 \\
\hline Amor & 213 & 9.62 & Cuidadosa & 308 & 15.68 \\
\hline Accesorios & 211 & 9.53 & Apariencia & 274 & 13.95 \\
\hline Actitud & 191 & 8.63 & Fuerza & 259 & 13.19 \\
\hline Respeto & 177 & 8.00 & Higiene & 248 & 12.63 \\
\hline Vanidad & 168 & 7.59 & Vanidad & 255 & 12.98 \\
\hline Cuidadosa & 136 & 6.15 & Actitud & 207 & 10.54 \\
\hline Madre & 132 & 5.96 & Rosa & 196 & 9.98 \\
\hline Pareja & 132 & 5.96 & Sensible & 190 & 9.67 \\
\hline Débil & 125 & 5.65 & Respeto & 165 & 8.40 \\
\hline Frágil & 122 & 5.51 & Elegancia & 147 & 7.48 \\
\hline TR & 136 & & TR & 164 & \\
\hline
\end{tabular}

Abrev.: $\mathbf{P S}=$ Peso semántico; $\mathbf{D S C}=$ Distancia semántica cuantitativa; $\mathbf{T R}=$ Tamaño de la red

Las primeras tres definidoras que aparecen sobre este concepto en ambos sexos son las de "mujer", "delicadeza" y "belleza". Femenino es definido por los hombres con palabras que hacen referen- cia a una mujer que necesita ser protegida por un varón: "débil" y "frágil", las que no aparecen en lo reportado por las mujeres y que hacen referencia a elementos propios de una relación de 
pareja dependiente, necesitada de protección y seguridad. Los conceptos ligados a la imagen, tales como "belleza", "cuidadosa", "accesorios" y "elegancia" representan para los jóvenes la exacerbación de la importancia de la apariencia y de mujeres atractivas que garanticen el éxito de una relación en su imaginario social. La mujer debe esforzarse por ser bella y adornarse de acuerdo al modelo vigente, lo que le otorga un valor que la coloca en un nivel de competencia con otras mujeres para acceder al "hombre ideal". Las palabras "madre" y "pareja" no aparecen en el núcleo de red de las mujeres, lo que representa una nueva asociación a proyectos propios desligados ya del "vivir para otros". Los hombres valoran atributos que refuerzan en las mujeres una feminidad de un modo tradicional, hegemónico, según el cual se espera que sean bellas, delicadas, dulces, sensibles y maternales.

Para el estímulo masculino los varones conformaron un total de 181 definidoras y las mujeres de 166. Dicho concepto fue definido por los participantes con las palabras "hombre" y "fuerza", principalmente. De entre las quince palabras descriptoras más mencionadas por hombres y mujeres se pueden observar semejanzas en $80 \%$ de ellas; es decir, ambos sexos definen masculino con atributos que socialmente se asignan a varones y que definen culturalmente la masculinidad, tales como "rudo", "apariencia", "poder" y "trabajo", entre otros, conceptos que denotan control y dominio de la persona (Tabla 4).

Tabla 4. Definidoras para el concepto masculino.

\begin{tabular}{|l|r|r|l|r|c|}
\hline \multirow{2}{*}{\multicolumn{1}{|c|}{ Definidora }} & \multicolumn{2}{c|}{ Hombres } & \multirow{2}{*}{ Definidora } & \multicolumn{2}{c|}{ Mujeres } \\
\cline { 2 - 3 } \cline { 6 - 7 } & PS & DSC (\%) & & \multicolumn{1}{c|}{ PS } & DSC (\%) \\
\hline Hombre & 1,836 & 100.00 & Fuerza & 2,131 & 100.00 \\
\hline Fuerza & 1,626 & 88.56 & Hombre & 1,989 & 93.34 \\
\hline Machismo & 490 & 26.69 & Machismo & 821 & 38.53 \\
\hline Rudo & 442 & 24.07 & Atractivo & 520 & 24.40 \\
\hline Apariencia & 264 & 14.38 & Apariencia & 435 & 20.41 \\
\hline Decisión & 198 & 10.78 & Caballerosidad & 259 & 12.15 \\
\hline Caballerosidad & 195 & 10.62 & Varonil & 247 & 11.59 \\
\hline Poder & 192 & 10.46 & Rudo & 207 & 9.71 \\
\hline Trabajo & 192 & 10.46 & Deportes & 189 & 8.87 \\
\hline Valor & 188 & 10.24 & Carácter & 178 & 8.35 \\
\hline Deportes & 168 & 9.15 & Poder & 164 & 7.70 \\
\hline Varonil & 165 & 8.99 & Sexo & 144 & 6.76 \\
\hline Respeto & 145 & 7.90 & Valor & 131 & 6.15 \\
\hline Sexo & 126 & 6.86 & Protección & 130 & 6.10 \\
\hline Comportamiento & 108 & 5.88 & Trabajo & 127 & 5.96 \\
\hline TR & 181 & & TR & 166 & \\
\hline
\end{tabular}

Abrev $:$ : PS $=$ Peso semántico, $\mathbf{D S C}=$ Distancia semántica cuantitativa, $\mathbf{T R}=$ Tamaño de la red.

Las primeras definidoras asociadas al concepto de masculinidad fueron palabras como "hombre", "fuerza", "machismo" y "apariencia", lo que habla de que los jóvenes responden a un modelo hegemónico en el que se exacerba la superioridad de los atributos masculinos sobre los que se recrea el poder. Aparecen de nuevo, al igual que con el concepto hombre, palabras asociadas con el trabajo productivo de los varones: "trabajo", "poder" y "valor". La selección hecha por los participantes alude a un éxito diferenciado para hombres y mujeres; en el caso de estas últimas, la importancia recae en el atractivo físico de una mujer: delicada, bella, dulce, cuidadosa, en tanto que de los hombres se espera la fuerza, el carácter, el poder, construidos sobre habilidades racionales. Algunos estudios plantean que la masculinidad requiere reafirmarse constantemente mediante este tipo de características socialmente valoradas (Connell, 1997; Kaufman, 1997). 
En síntesis, se observa que los participantes afirman que los varones son los proveedores y protectores de la familia, estereotipo que se deriva de la socialización del género. Aparecen además las palabras "fuerza", "rudo", "deportes", que entrañan actividades de competencia física, lo que guarda relación con un modelo atlético de atractivo sexual y vencedor. La masculinidad hegemónica reproduce un mandato claro para los hombres: ser fuertes y no débiles como las mujeres. Fuertes en más de un sentido, pues la fuerza física masculina se articula simbólicamente con el poder, y este con el hombre. Tal construcción de los cuerpos origina cuotas de poder inequitativas para las mujeres. Bajo este supuesto, los hombres deberían de ser activos, fuertes, aptos para el trabajo pesado, para el mando. En el caso de las mujeres, el concepto les evoca palabras que hacen referencia al amor romántico: "atractivo", "apariencia", "caballerosidad" y "varonil".

\section{DISCUSIÓN}

Los participantes mostraron una postura tradicional en cuanto a lo que define o caracteriza a un hombre y a una mujer, así como a lo masculino y lo femenino. Las palabras asociadas dan muestra en su mayoría de lo que socialmente se espera de cada sexo. Para esta muestra, los esquemas que definen los sexos siguen las pautas que marca el modelo patriarcal, en el que al varón se le relaciona con aquellas características que denotan poder y las mujeres con aquellas que tienen que ver principalmente con la reproducción y la sumisión.

Los resultados para los conceptos de mujer y hombre muestran que los participantes los describen con palabras que denotan la transmisión de valores tradicionales asociados a lo que socialmente se espera de todos y cada uno de ellos, todo eso permeado por un modelo hegemónico según el cual el ser hombre se asocia con la fuerza y el trabajo, aspectos necesarios todavía en una sociedad de gran competitividad. En cambio, a la mujer se le sigue vinculando con atributos que tienen que ver con la belleza y la delicadeza, ambos ligados de algún modo a la procreación. Los resultados coinciden con lo encontrado en un estudio realizado con 163 universitarios de la Universidad Na- cional Autónoma de México (Petra et al., 2000), en el que los participantes asociaron la palabra hombre con "fuerza", "inteligencia" y "trabajo" y mujer con "belleza", "inteligencia" y "amor por los varones", a las que agregaron la palabra "ternura". Hombres y mujeres siguen representándose a sí mismos según los roles que la sociedad les ha imbuido y reforzado, aunque se muestra una cierta tendencia a dar a la mujer un lugar que no necesariamente está ligado a la procreación, pese a lo cual el hombre continúa teniendo que mostrar su fuerza como una forma de conducirse en la sociedad actual (Aguilar, Valdez, González y González, 2013).

Esta connotación de los conceptos asociados a las palabras mujer y hombre indica la transmisión de valores asignados a los géneros a partir de los modelos construidos hegemónicamente, lo que afecta particularmente las relaciones afectivas y de pareja (Lamas, 1996). Lo anterior ocurre todavía, aunque en las familias empiezan a observarse transformaciones que paulatinamente evolucionan hacia unas relaciones más igualitarias (Amurrio et al., 2008). Las citadas creencias son transmitidas por la familia y reforzadas por las instituciones educativas a través de la cultura que dicta "el deber ser" y "el deber hacer" para unos y otras.

Algunos estudios hechos en América Latina han mostrado que están surgiendo nuevos modelos de sexualidad femenina y masculina. Tal es el caso del estudio de Cáceres (1995) sobre la cultura del adolescente en Perú. En dicho trabajo se pidió a niños de ambos sexos que nombraran tipos de niñas de acuerdo a su comportamiento sexual. Los resultados mostraron que tanto hombres como mujeres generaron una variedad de nombres distintos que describían diferentes tipos de niñas: como las que toman la iniciativa y no permiten que los varones las controlen fácilmente, lo que sugiere nuevos modelos de sexualidad femenina. Por el contrario, el estudio no mostró evidencia de que hubiera una diversidad similar entre los varones. Los jóvenes dijeron que se es hombre o se es "maricón", lo que muestra que los roles masculinos parecen ser más rígidos que los femeninos.

Por otro lado, la investigación de Bergara, Riviere y Bacete (2008), llevada a cabo en España con estudiantes de secundaria, aporta datos significativos en relación a la vigencia, prevalencia y 
fortaleza de los estereotipos y roles de género. Al preguntárseles cómo son los hombres y cómo son las mujeres, ambos sexos coincidieron en mencionar lo que socialmente se espera de cada sexo en todos los grupos. Por un lado, señalaron que las mujeres son sensibles, histéricas, intuitivas, cariñosas, detallistas y miedosas, y que los hombres son arriesgados, violentos, deportistas, infieles, activos, brutos y despistados.

Los resultados expuestos en el presente estudio ofrecen evidencias de que aun cuando los participantes eran estudiantes universitarios, mantenían todavía significados ceñidos a las representaciones tradicionales y conservadoras dominantes en el contexto sonorense, que prioriza lo masculino. Los conceptos asociados a lo masculino y femenino no variaron en cuanto a lo socialmente esperado. El hecho de ser universitarios haría suponer que es una población más permeada por cambios sociales que impulsan la igualdad y equidad de género, sin embargo no es así. Habría que preguntarse si las acciones que lleva a cabo la universidad están reforzando lo que prevalece en el modelo dominante, en lugar de promover prácticas conducentes hacia la transformación de las mentalidades, resignificando con ello las nociones de masculinidad y feminidad.

Los significados atribuidos socialmente sobre el ser hombre o ser mujer tienden a construir realidades que perpetúan las actuales inequidades de género, donde las mujeres son las principales afectadas. El cambio se ha ido observando gradualmente en las generaciones más jóvenes, pero aún no termina de extenderse a toda la sociedad. Es por ello indispensable educar a las futuras generaciones a partir de la idea de equidad y deconstruyendo los estereotipos asociados a los hombres y a las mujeres.

\section{REFERENCIAS}

Aguilar, Y., Valdez, J., González, N. y González, S. (2013). Los roles de género de los hombres y las mujeres en el México contemporáneo. Enseñanza e Investigación en Psicología, 18(2), 207-224.

Amurrio, M., Larrinaga, A., Usategui, E. y Del Valle, A. (2008). Violencia de género en las relaciones de pareja de adolescentes y jóvenes de Bilbao. Conclusiones de Informe. Bilbao (España): Universidad del País Vasco y Ayuntamiento de Udala.

Bergara, A., Riviere, J. y Bacete, R (2008). Los hombres, la igualdad y las nuevas masculinidades. Vitoria-Gasteiz (España): Emakunde-Instituto Vasco de la Mujer. Recuperado de http://www.aulaviolenciadegeneroenlocal.es/consejosescolares/archivos/P_013_los_hombres_la_igualdad.pdf.

Cáceres, C. (1995). Bisexualidades masculinas en la Lima de los noventa: consideraciones de salud sexual. En El amor y sus especies. Primer Seminario del Archivo Etnográfico Audiovisual: Herramientas para la catalogación, conservación y digitalización de material fotográfico. Lima: PUCP.

Cochran, W. (1990). Técnicas de muestreo. México: CECSA.

Connell, R. (1997). La organización social de la masculinidad. En T. Valdez y J. Olavarría (Eds.): Masculinidad/es: Poder y crisis (pp. 31-48). Santiago de Chile: ISIS-FLACSO.

Díaz-Guerrero, R. (1989). Una etnopsicología mexicana. Ciencia y Desarrollo, 15(86), 69-85.

Figueroa, J., González, E. y Solís, V. (1981). Una aproximación al problema del significado: las redes semánticas. Revista Latinoamericana de Psicología, 13(3), 447-458.

Instituto Nacional de Estadística y Geografía (2013). Estadísticas a propósito del Día de la Juventud: Datos de Sonora. Aguascalientes (México): INEGI.

Kaufman, M. (1997). Las experiencias contradictorias del poder entre los hombres. En T. Valdés y J. Olavarría (Eds.): Masculinidad/es. Poder y crisis (pp.63-81). Santiago de Chile: ISIS-FLACSO.

Lamas, M. (1996). El género: la construcción social de la diferencia sexual. México: Porrúa.

Lamas, M. (2002). Usos, dificultades y posibilidades de la categoría "género". En M. Lamas (Comp.): Cuerpo: diferencia sexual y género. México: Taurus.

Lara-Cantú, M. (1993). Inventario de masculinidad y femineidad. México: IMAFE.

Maldonado, I. y Segovia, A. (2010). Premisas culturales que sostienen relaciones no satisfactorias. En S. Lerner y L. Melgar (Eds.): Familias en el siglo XXI: Realidades diversas y políticas públicas (pp. 229-244). México: PUEG-UNAM/COLMEX/El Manual Moderno.

Núñez, G. (2013). Hombres sonorenses. Un estudio de género en tres generaciones. México: Universidad de Sonora/Pearson Educación, México. 
Ortiz H., L. (2004). La opresión de minorías sexuales desde la inequidad de género. Política y Cultura, 22, 161-182.

Petra, I., Talayero, J., Fouilloux, C. y Díaz, A. (2000). Conceptualización semántica de los términos "hombre” y “mujer" por estudiantes del primer año de Medicina. Revista de la Facultad de Medicina, 43(5), 185-191.

Reyes L., I. (1993). Redes semánticas para la construcción de instrumentos. Revista de Psicología Social y Personalidad, 9(1), 83-99.

Rocha S., T. y Díaz-Loving, R. (2005). Cultura de género: La brecha ideológica entre hombres y mujeres. Anales de Psicología, $21(1), 42-49$.

Scott, J. (2003). El género. La construcción cultural de la diferencia sexual. México: PUEG.

Valdez M., J.L. (2002). Las redes semánticas naturales: usos y aplicaciones en psicología social. Toluca (México): Universidad Autónoma del Estado de México.

Varela, V. (2012). Estudio sobre el concepto que tiene de la mujer moderna: tres muestras no correlacionadas. Revista Iberoamericana de Psicología: Ciencia y Tecnología, 5(2), 77-83. 